


\section{BEIHEFTE}

ZUR

\section{ZEITSCHRIFT \\ FUR \\ ROMANISCHE PHILOLOGIE}

BEGRÜNDET VON Prof. Dr. GUSTAV GRÖBER †

FORTGEFUHRT UND HERAUSGFGEBEN

rov

DR. ERNST HOEPFFNER

PKOFKSSOK AN DRR UNIVERSITÄT JENA

LII. HEFT

ERICH NIESTROY

DER TROBADOR PISTOLETA

FRITZ NAUDIETH

DER TROBADOR GUILLEM MAGRET

HALLE A.S.

VERLAG VON MAX NIEMEYER

1914 


\title{
DER \\ TROBADOR PISTOLETA
}

\author{
HERAUSGEGEBEN \\ vor \\ ERICH NIESTROY \\ DER TROBADOR \\ GUILLEM MAGRET \\ HERAUSGEGEBEN \\ voN
}

FRITZ NAUDIETH

HALLE A.S.

VERLAG VON MAX NIEMEYER

1914 



\section{Herrn Professor Dr. Alfred Pillet in dankbarer Verehrung gewidmet von}

Erich Niestroy und Fritz Naudieth 
